\title{
Delivering high value therapies in COPD: the secret is in the marketing
}

\author{
Helen Ashdown ${ }^{1}$ and Michael Steiner ${ }^{2}$ \\ Affiliations: ${ }^{1}$ Nuffield Dept of Primary Care Health Sciences, University of Oxford, Radcliffe Observatory \\ Quarter, Oxford, UK. ${ }^{2}$ Institute for Lung Health, Leicester Biomedical Research Centre - Respiratory, \\ University of Leicester, Glenfield Hospital, Leicester, UK.
}

Correspondence: Michael Steiner, Institute for Lung Health, Leicester Biomedical Research Centre Respiratory, University of Leicester, Glenfield Hospital, Groby Road, Leicester, LE3 9QP, UK.

E-mail: ms346ale.ac.uk

@ERSpublications

The importance of prioritising uptake of high value, non-pharmacological therapies in the management of COPD http://ow.ly/AA2u30ol4t5

Cite this article as: Ashdown H, Steiner M. Delivering high value therapies in COPD: the secret is in the marketing. Eur Respir J 2019; 53: 1900215 [https://doi.org/10.1183/13993003.00215-2019].

"Content is King but Engagement is Queen and the Lady Rules the House"

- Mari Smith, Facebook marketing guru.

There are a wide range of therapies now available for people suffering with chronic obstructive pulmonary disease (COPD), both pharmacological and non-pharmacological [1]. However, there has been recent recognition that non-pharmacological approaches (for example smoking cessation and pulmonary rehabilitation) deliver the highest value in terms of patient benefit in relation to cost [2]. It is also evident that the provision of high value therapies in clinical practice is frequently sub-optimal, as illustrated in figure 1 summarising data from a recent, comprehensive primary care audit of COPD care in Wales [3]. In healthcare systems such as Australia and the UK, where $90 \%$ of healthcare is delivered in primary care [4], there is now a need to investigate how best to implement such high value interventions in primary care.

In this issue of the European Respiratory Journal, LIANG et al. [5] present the results of a cluster randomised trial undertaken in primary care in Australia which investigated the effectiveness of an interdisciplinary bundle of interventions, comprising smoking cessation support, home medicines review by a pharmacist, and a home-based pulmonary rehabilitation programme (termed the "RADICALS model of care"). They used a case-finding approach to identify potential patients based on a smoking history on general practitioner (GP)'s records who, along with those with an existing coded diagnosis, were then eligible for the trial if baseline spirometry fulfilled diagnostic criteria for COPD. In practices allocated to the intervention group, patients were invited by the research team (working with clinical practice staff) to take part in the three components of the intervention. The study failed to demonstrate significant benefits at 6 or 12 months in health status (the primary outcome measure) or other outcomes, including smoking abstinence, symptom scores, lung function or psychological status, in those allocated to the RADICALS intervention. This was probably largely due to poor uptake as $43 \%$ patients in the intervention group did not receive any part of the intervention. In line with this, the per-protocol analysis of those completing the full intervention found a clinical and statistically significant improvement in St George's Respiratory Questionnaire Score (difference 5.22 (95\% CI 0.19 to 10.25), although no improvement was found in any secondary outcomes. 
a)

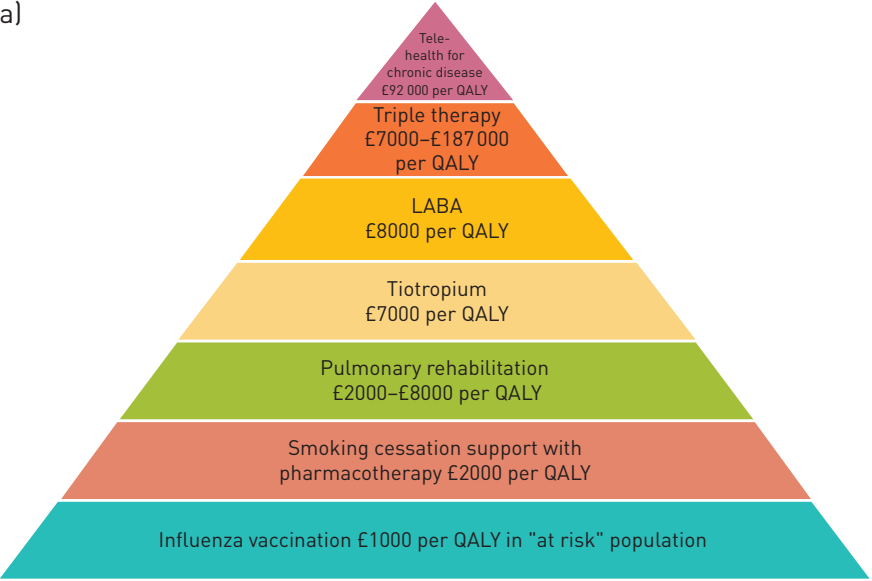

b)

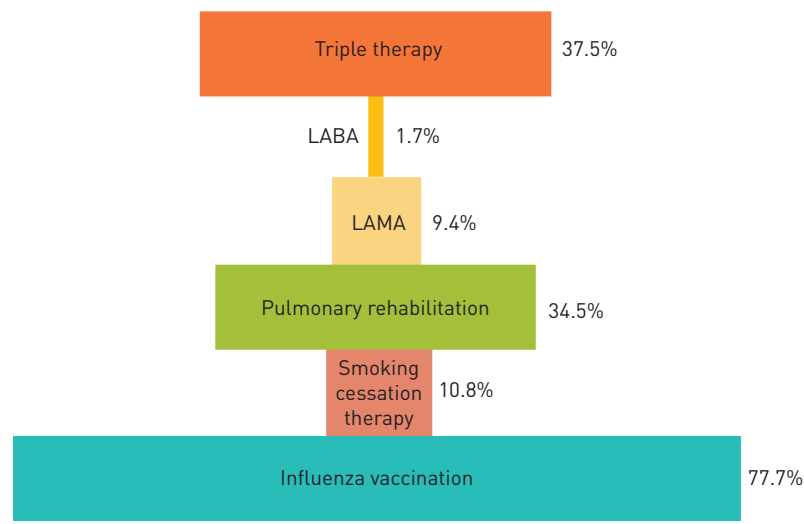

FIGURE 1 a) The chronic obstructive pulmonary disease (COPD) value pyramid is a model designed to aid clinicians and providers to make value-based decisions for people and populations with COPD, through assessing the comparative value of interventions in terms of cost per individual per quality-adjusted life year (QALY). Reproduced with permission of the London Respiratory Network 2013 [2]. b) The equivalent value pyramid providing a representation of the proportion of people who were receiving value-based interventions for COPD in primary care in Wales in 2014-2015. Reproduced with permission from the National Asthma and COPD Audit Programme (NACAP), Royal College of Physicians [3]. LAMA: long-acting muscarinic antagonist; LABA: long-acting beta agonist.

Despite its overall negative findings, such a well designed and conducted trial provides a number of important lessons about the barriers to early and accurate diagnosis of COPD and the delivery of value interventions in the primary care setting. The study participants could be described as an "at risk" population with relatively mild disease as there was a high prevalence of active smoking (50-70\%) but symptom scores and self reported breathlessness were low and lung function impairment was mild. A substantial number of patients (nearly half) had not previously been diagnosed with COPD despite attending for appointments with the practice on at least two occasions in the preceding year. This is in line with other studies suggesting potential delays in diagnosis of COPD in patients managed in primary care [6]. We don't know the reasons for these previous consultations but it is quite likely for many patients that the content of the RADICALS intervention did not address the problems they perceived as important for their health (indeed, those who took up the intervention were those with more severe disease and lower SGRQ scores). Likewise, GPs were relied on to refer patients to the component interventions in addition to providing usual care. Workload in primary care has increased dramatically in recent years (a UK study estimates a 16\% increase in workload [7]) and it is possible that at times GPs just had too many competing demands for their time for this to be a priority for them or their patient.

In many respects, the finding of the study is in keeping with the experience of implementation of therapies requiring a component of behaviour change in patients. Smoking cessation success rates were low but of similar magnitude to general success rates for brief smoking cessation interventions [8]. Likewise, uptake of pulmonary rehabilitation, itself the exemplar inter-disciplinary intervention in COPD, is low in clinical practice both in primary and secondary care [9]. The problem is not restricted to COPD; in the UK uptake of structured education programmes for type II diabetes is $9 \%$ and for weight loss interventions in obesity $9 \%[10,11]$. Given the large magnitude of benefits delivered by these non-pharmacological interventions, small increments in the numbers of patients taking them up may result in substantial health benefits and there is a pressing need to develop methodologies to enhance patient uptake. The experience of the Ottawa model of smoking cessation suggests that more proactive/ intensive support has the potential to achieve this [12]. There is evidence also that the specifics of the interaction between doctor and patient impacts on how likely the patient is to take up the referral [13], so research and training on communication may also increase uptake of such interventions. There is also a risk that patients undervalue complex but highly efficacious non-pharmacological measures such as pulmonary rehabilitation or smoking cessation support because of the delay in accessing these compared with pharmacological therapies, which can be prescribed on the spot.

Other studies testing the effectiveness of bundled or "integrated" multi-component interventions in COPD in primary care have similarly shown variable impact on health outcomes $[14,15]$. As the authors of the RADICALS trial point out, these studies are difficult to summarise because of wide variations in the content of the interventions and indeed the target population as some were focused on enhancing the knowledge and training of practice clinical staff rather than directly delivering therapy to patients. As with the RADICALS trial, many aimed to improve health outcomes (such as health status or hospitalisation 
rates) but most comprised therapies already established as effective in previous trials (such as pulmonary rehabilitation). We suggest that the focus of attention should shift towards quantifying uptake of therapies as the primary objective rather than clinical outcomes that, based on the established scientific knowledge, we can be confident will follow if this is achieved.

The difficulties experienced by the researchers implementing the RADICALS intervention provides pointers to how to overcome barriers to the implementation of high value, non-pharmacological therapies in real world clinical practice settings. We suggest the uniform systemised approach taken here (and inherent in clinical trial design) will ultimately limit patient acceptance and will need to be allied to individual patient problem-solving based around the clinical problems patients bring into the consulting room [16].

However personalised the design of any proposed intervention is, the degree of an individual patient's understanding and engagement will always be a key limitation. Such "activation" can be quantified with validated instruments and should be a consideration in assessing effectiveness [17]. Marrying this with efforts to enhance the knowledge and skills of clinical teams might offer a path to delivering meaningful increments in uptake. This is encapsulated in the Chronic Care Model which proposes the organisation of care around the support for a productive interaction between an activated patient and a prepared clinical team [18]. There is evidence that if the components of the Chronic Care Model are implemented in this way, health benefits accrue in long term conditions including COPD [19].

The RADICALS trial offers reasons to be cheerful: there is much that can be offered to patients with COPD if this offer is appropriately marketed to clinical need.

Conflict of interest: H. Ashdown reports consultancy fees paid to institution from Boehringer Ingelheim, outside the submitted work. M. Steiner reports personal fees for advisory board work, honoraria for speaking engagements, and travel support for meeting attendance from Boehringer Ingelheim and GSK, personal fees for advisory board work from Nutricia, outside the submitted work.

\section{References}

1 Vogelmeier CF, Criner GJ, Martinez FJ, et al. Global Strategy for the Diagnosis, Management, and Prevention of Chronic Obstructive Lung Disease 2017 Report. GOLD Executive Summary. Am J Respir Crit Care Med 2017; 195: 557-582.

2 Williams S, Baxter N, Holmes S, et al. IMPRESS Guide to the Relative Value of COPD Interventions. British Thoracic Society/Primary Care Respiratory Society UK, 2012.

3 Baxter N, Holzhauer-Barrie J, McMillan V, et al. Time to Take a Breath. National Chronic Obstructive Pulmonary Disease (COPD) Audit Programme: Clinical Audit of COPD in Primary Care in Wales 2014-15. London, Royal College of Physicians, 2016.

4 NHS England. Transforming Primary Care in London. General Practice. A Call to Action. London, National Health Service, 2013.

5 Liang J, Abramson MJ, Russell G, et al. Interdisciplinary COPD intervention in primary care: a cluster randomised controlled trial. Eur Respir J 2019; 53: 1801530.

6 Jones RC, Price D, Ryan D, et al. Opportunities to diagnose chronic obstructive pulmonary disease in routine care in the UK: a retrospective study of a clinical cohort. Lancet Respir Med 2014; 2: 267-276.

7 Hobbs FDR, Bankhead C, Mukhtar T, et al. Clinical workload in UK primary care: a retrospective analysis of 100 million consultations in England, 2007-14. Lancet 2016; 387: 2323-2330.

8 Royal College of Physicians. Hiding in Plain Sight: Treating Tobacco Dependency in the NHS. London, Royal College of Physicians, 2018.

9 Steiner M, McMillan V, Lowe D, et al. Pulmonary rehabilitation: An exercise in improvement. National Chronic Obstructive Pulmonary Disease (COPD) Audit Programme: Clinical and organisational audits of pulmonary rehabilitation services in England and Wales 2017. National report. London, Royal College of Physicians, 2018.

10 Aveyard P, Lewis A, Tearne S, et al. Screening and brief intervention for obesity in primary care: a parallel, two-arm, randomised trial. Lancet 2016; 388: 2492-2500.

11 UK National Diabetes Audit: NHS Digital. National Diabetes Audit Report 1: Findings and Recommendations 2016-17. Health and Social Care Information Centre, 2018.

12 Papadakis S, Cole AG, Reid RD, et al. Increasing rates of tobacco treatment delivery in primary care practice: evaluation of the Ottawa model for smoking cessation. Ann Fam Med 2016; 14: 235-243.

13 Albury C, Stokoe E, Ziebland S, et al. GP-delivered brief weight loss interventions: a cohort study of patient responses and subsequent actions, using conversation analysis in UK primary care. Br J Gen Pract 2018; 68: e646-e653.

14 Kruis AL, Boland MR, Assendelft WJ, et al. Effectiveness of integrated disease management for primary care chronic obstructive pulmonary disease patients: results of cluster randomised trial. BMJ 2014; 349: g5392.

15 Kruis AL, Smidt N, Assendelft WJ, et al. Integrated disease management interventions for patients with chronic obstructive pulmonary disease. Cochrane Database Syst Rev 2013: CD009437.

16 Steiner MC, Evans RA, Greening NJ, et al. Comprehensive respiratory assessment in advanced COPD: a 'campus to clinic' translational framework. Thorax 2015; 70: 805-808.

17 Hibbard JH, Stockard J, Mahoney ER, et al. Development of the Patient Activation Measure (PAM): conceptualizing and measuring activation in patients and consumers. Health Serv Res 2004; 39: 1005-1026.

18 Bodenheimer T, Wagner EH, Grumbach K. Improving primary care for patients with chronic illness: the chronic care model, Part 2. JAMA 2002; 288: 1909-1914.

19 Adams SG, Smith PK, Allan PF, et al. Systematic review of the chronic care model in chronic obstructive pulmonary disease prevention and management. Arch Intern Med 2007; 167: 551-561. 